Physics, Chemistry, and Dynamics of Interplanetary Dust

ASP Conference Series, Vol. 104, 1996

Bo A. S. Gustafson and Martha S. Hanner (eds.)

\title{
Laboratory Experiments on Carbonaceous and Silicate Materials
}

\author{
L. Colangeli, V. Mennella and A. Rotundi
}

Astronomical Observatory of Capodimonte, Via Moiariello 16, 80131 Napoli (Italy)

E. Bussoletti and P. Palumbo

Istituto Universitario Navale, Via A. De Gasperi 5, 80133 Napoli (Italy)

\begin{abstract}
Ground-based, remote and "in situ" observations show that dust plays an important role in the evolution of interstellar, circumstellar and interplanetary media. Despite the wealth of available astronomical data, a main item to be clarified concerns the actual chemico-physical composition of cosmic dust. A correct interpretation of observations must rely on a systematic comparison with results coming from laboratory simulations. In this paper we present an overview of recent results, obtained in our laboratory, on various forms of carbon-based and silicate grains of possible astrophysical interest.
\end{abstract}

\section{Introduction}

Several experimental lines are available to get information about solid materials in space. Astronomical observations of interstellar and circumstellar environments provide a wide variety of spectroscopic data which contain hints about composition, formation and evolution of grains. Studies of planetary bodies are a further source of information: remote and "in situ" measurements on comets are useful to analyse similarities and/or differences between interstellar and interplanetary media. Laboratory experiments on meteorites and interplanetary dust particles allow us to monitor evolutionary traces in cosmic materials.

Similar spectral features may suggest relations between materials observed in different space environments. For example, the UV interstellar and circumstellar extinction "bumps", falling around 220 and $240-250 \mathrm{~nm}$ respectively (e.g. Fitzpatrick \& Massa 1988; Buss et al. 1989), are attributed to carbon-based materials; the 3.3-3.4 $\mu \mathrm{m}$ bands, observed in absorption or emission in molecular clouds, toward the galactic center, in Wolf-Rayet stars and in comets (e.g. Tokunaga \& Brooke 1990), are a clear indication of $\mathrm{C}-\mathrm{H}$ stretching resonances in molecular and/or solid compounds; the absorption/emission 10 and $20 \mu \mathrm{m}$ bands, present in the spectra of circumstellar envelopes, dark clouds and comets (e.g. Hanner et al. 1994; 1995), are the clear signature of silicate grains. A simple spectral identification of a material's fingerprint is not sufficient to completely understand how grains behave in different space conditions. Laboratory experiments, performed by several research groups (see, e.g., the Proceedings of 
the Conference "Dust, molecules and backgrounds: from laboratory to space", Bussoletti et al. 1995), are aimed at simulating formation and evolution of grains and provide a powerful tool to interpret astronomical data on the basis of the information gained on cosmic dust "analogue" samples.

In section 2 we will provide schematic information about production methods and analytical techniques used in our laboratory to study carbonaceous and silicate analogue samples. Section 3 will be devoted to present our most significant results, while in section 4 we will discuss the relevance of our data to the interpretation of astronomical observations concerning cosmic dust.

\section{Laboratory experiments}

The development of laboratory experiments on cosmic dust analogue materials can be summarised in the following steps:

1. production of carbonaceous and silicate grains, differentiated in morphological, chemical and optical properties;

2. study of the materials by means of various techniques which are able to provide a complete characterisation of morphology, structure, chemical and optical properties as a function of production and treatment parameters;

3. interpretation of obtained results in a self-consistent scenario that has a general validity and can be extrapolated also to cosmic dust conditions.

Table 1. Grain condensation techniques.

\begin{tabular}{|c|c|c|c|c|c|c|c|}
\hline \multirow[t]{2}{*}{ Sample } & \multirow[t]{2}{*}{ Raw material } & \multirow[t]{2}{*}{ Production method } & \multicolumn{5}{|c|}{ Production conditions $a$} \\
\hline & & & Atm. & $P$ & $d$ & $V$ & $I$ \\
\hline$\overline{\mathrm{ACAR}}$ & Amorphous carbon & Arc discharge & $\overline{\mathrm{Ar}}$ & 10 & 5 & $\overline{100}$ & 10 \\
\hline $\mathrm{ACH} 2$ & Amorphous carbon & Arc discharge & $\mathrm{H}_{2}$ & 10 & 5 & 100 & 10 \\
\hline $\mathrm{BE}$ & Benzene & Burning & air & 10 & 5 & - & - \\
\hline & & & & & & $R_{1}$ & $R_{2}^{b}$ \\
\hline CLI1 ${ }^{c}$ & Premixed reagents & Laser bombardment & $\overline{\mathrm{O}_{2}}$ & 10 & 5 & 2.0 & 1.5 \\
\hline $\mathrm{OLI} 2^{c}$ & Premixed reagents & Laser bombardment & $\mathrm{O}_{2}$ & 10 & 5 & 2.0 & 0.1 \\
\hline $\operatorname{COSMIC}^{d}$ & Premixed reagents & Laser bombardment & $\mathrm{O}_{2}$ & 10 & 5 & 2.0 & 1.5 \\
\hline ENST $^{e}$ & Ground natural silicate & Laser bombardment & $\mathrm{O}_{2}$ & 10 & 5 & 1.0 & 0.1 \\
\hline SERPe & Ground natural silicate & Laser bombardment & $\mathrm{O}_{2}$ & 10 & 5 & 1.5 & 0.0 \\
\hline
\end{tabular}

${ }^{a}$ Atm.: ambient gas; $V, I$ : applied voltage and current during the discharge; $d$ : collecting distance

${ }^{b} R_{1}=$ metal $/ \mathrm{Si}$ ratio; $R_{2}=\mathrm{Fe} / \mathrm{Mg}$ ratio

${ }^{c} \mathrm{OLI} 1=\left(\mathrm{Fe}_{0.6} \mathrm{Mg}_{0.4}\right)_{2} \mathrm{SiO}_{4} ; \mathrm{OLI} 2=\left(\mathrm{Fe}_{0.1} \mathrm{Mg}_{0.9}\right)_{2} \mathrm{SiO}_{4}$

${ }^{d} \mathrm{COSMIC}=\left(\mathrm{Fe}_{0.6} \mathrm{Mg}_{0.4} \mathrm{Al}_{0.035} \mathrm{Ca}_{0.03} \mathrm{Na}_{0.025}\right)_{2} \mathrm{SiO}_{4}$

${ }^{e}$ ENST $=\left(\mathrm{Mg}_{0.9} \mathrm{Fe}_{0.1}\right) \mathrm{SiO}_{3} ; \mathrm{SERP}=\left(\mathrm{Mg}_{6} \mathrm{Si}_{4} \mathrm{O}_{10}\right)(\mathrm{OH})_{8}$

In Table 1 we summarise the condensation techniques and boundary conditions used in our experiments to produce analogue samples. These methods are extensively presented in previous papers (e.g. Colangeli et al. 1995; Stephens 
et al. 1995), to which we refer the reader for further details. Several techniques are applied to characterise at best the dust products of our experiments. The used devices and the kind of information we have obtained are listed in Table 2.

We recall that ACAR and ACH2 samples are obtained by striking an arc between amorphous carbon electrodes in argon and in $\mathrm{H}_{2}$ atmosphere, respectively; $\mathrm{BE}$ grains are synthesised by burning benzene in air. In addition, the changes experienced by $\mathrm{ACH} 2$ samples after thermal annealing, for 3 hours at $P<10^{-5}$ mbar, at different temperature steps up to $800{ }^{\circ} \mathrm{C}$ have been studied (Mennella et al. 1995a,b).

Olivine-type silicates are condensed by bombarding a target of previously mixed reagents by an excimer laser beam (see also Blanco et al. 1991). The samples considered here are characterised by the same metal $/ \mathrm{Si}$ ratio, $R_{1}=$ 2 , but have different $\mathrm{Fe} / \mathrm{Mg}$ ratio $R_{2}$ (see Table 1). Ground natural enstatite, ENST $=\left[\left(\mathrm{Mg}_{0.9} \mathrm{Fe}_{0.1}\right) \mathrm{SiO}_{3}\right]$, and serpentine, SERP $=\left[\left(\mathrm{Mg}_{6} \mathrm{Si}_{4} \mathrm{O}_{10}\right)(\mathrm{OH})_{8}\right]$, have been processed by laser bombardment, for comparison. We note that the $R_{1}$ and $R_{2}$ values reported in Table 1 refer to the "nominal" ratios of the starting compounds. However, our preparation methods may significantly affect the elemental relative abundances of the final dust products. For example, heating of SERP vaporises $\mathrm{H}_{2} \mathrm{O}$ so that $\mathrm{Mg}(\mathrm{OH}, \mathrm{O})_{6}$ octahedra are destroyed and the sample becomes a m:xture of olivine- and enstatite-type silicates. On the other hand, the laser-processing in an oxidising atmosphere may transform a considerable part of $\mathrm{Fe}^{2+}$ to $\mathrm{Fe}^{3+}$; these last cations can substitute $\mathrm{Si}$ in the tetrahedra and the $\mathrm{Fe} / \mathrm{Mg}$ ratio can be drastically reduced by this effect. Despite of this, preliminary EDX (Energy Dispersive X-ray) analyses of the dust products provide an elemental composition consistent with the starting ratios within about $10 \%$.

Table 2. Devices used to characterise samples.

\begin{tabular}{ll} 
Technique & Information \\
\hline Transmission/scanning electron microscopy & Shape, size, aggregation of grains \\
Electron diffraction & Structure \\
Raman spectroscopy & Structure \\
Energy Dispersive X-ray analysis & Elemental composition, purity \\
Spectroscopy $(40 \mathrm{~nm}-2 \mathrm{~mm})$ & Optical properties (EUV-mm), optical gap \\
\hline
\end{tabular}

\section{Results}

The morphological properties of the samples considered here, as deduced by transmission (T.E.M.) and scanning (S.E.M.) electron microscopy analyses, can be summarised as follows:

1. ACAR and ACH2 grains are spheroidal with average diameter of about 11-12 $\mathrm{nm}$; grains are agglomerated in clusters of some 4-5 particles, which, in turn, form chain structures;

2. BE grains have similar properties, but the average diameter is $30 \mathrm{~nm}$; 
3. silicate samples are formed by tangled strings of grains with average diameter of about $30 \mathrm{~nm}$.

As far as the optical properties are concerned, in Figure 1 we report the extinction measured for carbon-based grains (see also Colangeli et al. 1995; Mennella et al. 1995a,b). We note that all the spectra are characterised by:

1. a far UV maximum which falls off around $80-90 \mathrm{~nm}$;

2. a UV bump between 200 and $300 \mathrm{~nm}$, whose exact position depends upon production conditions and annealing of the samples; the peak is lacking in the as-produced ACH2 sample;

3. a decrease of extinction with increasing wavelength from the visual to the $\mathrm{mm}$ range, which can be fitted by a power law, $\propto \lambda^{-\alpha}$, with power index $\alpha$ varying between 1.3 and 0.3 , depending on the kind of sample and spectral sub-interval;

4. a number of IR features, mainly due to $\mathrm{C}-\mathrm{H}$ and $\mathrm{C}-\mathrm{C}$ resonances.

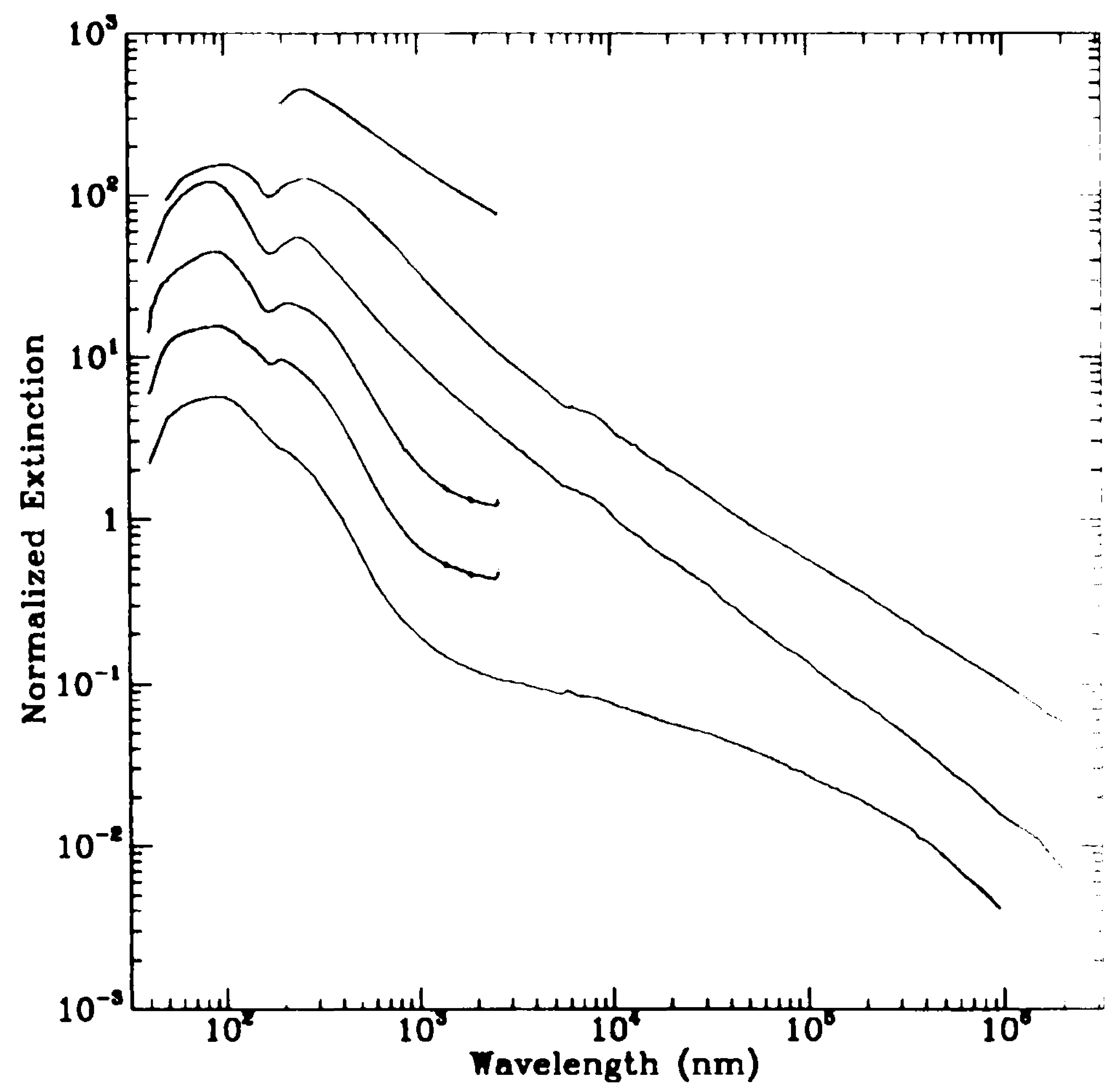

Figure 1. Extinction of amorphous carbon grains. From bottom to top curves refer to: $\mathrm{ACH} 2, \mathrm{ACH} 2$ annealed at $250^{\circ} \mathrm{C}, \mathrm{ACH} 2$ annealed at $415^{\circ} \mathrm{C}, \mathrm{ACAR}, \mathrm{BE}$ and $\mathrm{ACH} 2$ annealed at $800^{\circ} \mathrm{C}$. Curves are arbitrarily shifted in ordinate.

For silicate grains, the most important part of the spectrum concerns the $\mathrm{Si}-\mathrm{O}$ stretching and $\mathrm{O}-\mathrm{Si}-\mathrm{O}$ bending bands at 10 and $20 \mu m$, respectively; they are reported in Figure $2(\mathrm{a}, \mathrm{b})$.

\section{Discussion and astrophysical considerations}

For both $\mathrm{C}$-based and $\mathrm{Si}$-based grains we have reported here a set of laboratory data on a variety of samples, whose properties differ due to the production 


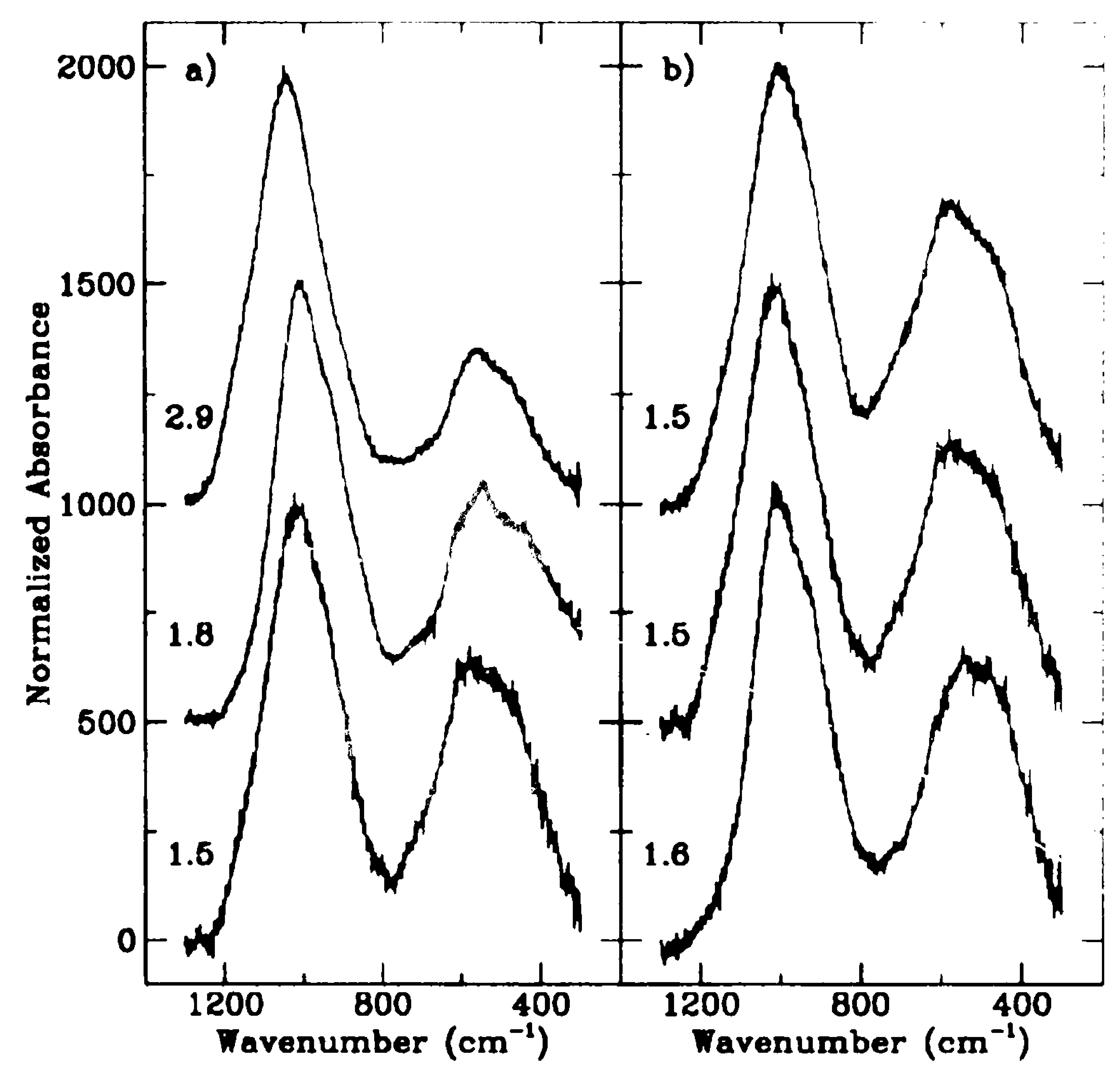

Figure 2. Absorbance spectra of silicate grains in the 10-20 $\mu \mathrm{m}$ band region. The spectra of ENST $\left(R_{1}=1.0\right)$, SERP $\left(R_{1}=1.5\right)$ and OLI1 $\left(R_{1}=2.0\right)$ are reported from top to bottom in panel a), while the spectra of COSMIC silicate $\left(R_{2}=1.5\right)$, OLI2 $\left(R_{2}=1.5\right)$ and OLI1 $\left(R_{2}=0.1\right)$ are shown from top to bottom in panel $b$ ). Spectra are arbitrarily shifted in ordinate. Numbers beside each curve refer to the $10 \mu \mathrm{m} / 20 \mu \mathrm{m}$ intensity ratio.

conditions and/or to the applied processing. A fundamental goal is to interpret these data in a systematic way and to relate them to boundary parameters during condensation and treatment. Actually, the differences measured among the carbon grain types can be interpreted taking into account variations of a structural parameter: the coherence length, $L_{a}$, of aromatic clusters forming the single particles, which are amorphous on a grain-size scale. Elements in favour of this interpretation are the variations measured as a function of thermal annealing of $\mathrm{ACH} 2$ samples and going from ACAR to BE samples (see Mennella et al. 1995a,b for more details): a) the progressive reduction of hydrogen content, the shift of the UV peak (attributed to $\pi-\pi^{*}$ electronic transitions) towards $260 \mathrm{~nm}$ and the closing of the energy gap; b) the inversion of the $3.3 \mu \mathrm{m} / 3.4$ $\mu m$ intensity ratio; $c)$ the change of intensity ratio for the so-called "diamondlike" (at $1310 \mathrm{~cm}^{-1}$ ) and "graphite-like" (at $1590 \mathrm{~cm}^{-1}$ ) Raman bands (see Mennella et al. 1995b). We conclude that, as long as the thermal annealing of $\mathrm{ACH} 2$ grains proceeds or, similarly, going from ACH2 to ACAR and to BE samples, a dimensional growth of the $\mathrm{sp}^{2}$ clusters forming grains occurs, i.e. a progressive transformation from aliphatic to aromatic character.

The spectra reported in Figure 2(a,b) for silicate grains show a progressive change of feature profile according to the detailed chemical composition of the species (see Stephens et al. 1995 for more details). In fact, according to the spectra in Figure 2a, as the ratio $R_{1}=$ metal/Si increases: a) the $10 \mu \mathrm{m}$ peak position shifts towards longer wavelengths; b) the $10 \mu \mathrm{m} / 20 \mu \mathrm{m}$ intensity ratio 
decreases. On the other hand, the ratio $R_{2}=\mathrm{Fe} / \mathrm{Mg}$ mainly affects the position of the $20 \mu \mathrm{m}$ band (see Figure $2 \mathrm{~b}$ ). We conclude that the $R_{1}$ and $R_{2}$ parameters tune the detailed spectral properties of silicates.

Although we do not claim that our samples are actual duplicates of cosmic dust materials, we believe that the physical and chemical parameters which determine the optical behaviour of our analogues may be efficient also for $\mathrm{C}-$ and $\mathrm{Si}$-based materials present in space. So far, our experiments have clearly demonstrated the relevance of three parameters: $L_{a}$, for carbons, and $R_{1}$ and $R_{2}$, for silicates. According to our interpretation, we expect that carbonaceous interstellar grains, responsible for the UV extinction bump at $220 \mathrm{~nm}$, are less graphitic (i.e. have smaller $L_{a}$ ) than grains responsible for the $240-250 \mathrm{~nm}$ circumstellar bump. Similarly, in the space environments where the typical IR C-H stretching bands are observed, dominant 3.4-3.5 $\mu \mathrm{m}$ bands indicate an aliphatic character, while a relevant $3.3 \mu \mathrm{m}$ band suggests a mainly aromatic (i.e. more graphitic) structure of carriers. The identification of cosmic silicate compounds still appears far from complete. Actually, an interpretation of available astronomical observations in terms of $R_{1}$ and $R_{2}$ parameters may result in a more systematic classification of spectra measured so far.

In conclusion, the laboratory results reported in the present work clearly show that experiments on cosmic dust analogues are crucial to identify connections between dust types present in different cosmic environments.

Acknowledgments. This work was supported by ASI, CNR and MURST. We thank A. Blanco, S. Fonti and J.R. Stephens for fruitful discussion on experimental data interpretation. S. Inarta, N. Staiano and E. Zona are thanked for technical assistance during experimental activity.

\section{References}

Blanco, A., Bussoletti, E., Colangeli, L., Fonti, S., Orofino, V., \& Stephens, J. R. 1991, in Origin and Evolution of Interplanetary Dust, A. C. LevasseurRegourd \& H. Hasegawa, Dordrecht: Kluwer, 125

Buss, R.H., Lamers, H., \& Snow, T.P. 1989, ApJ, 347, 977

Bussoletti, E., Colangeli, L., \& Mennella, V. Eds. 1995, Planet Spa. Sci., Vol. 43.

Colangeli, L., Mennella, V., Palumbo, P., Rotundi, A., \& Bussoletti, E. 1995, $A \mathscr{E} A S, \mathbf{1 1 3}, 561$

Fitzpatrick, E. L., \& Massa, D. 1988, ApJ, 328, 734

Hanner, M. S., Lynch, D. K., \& Russell, R. W. 1994, ApJ, 425, 274

Hanner, M. S., Brooke, T. Y., \& Tokunanga, A. T. 1995, ApJ, 438, 250

Mennella, V., Colangeli, L., Blanco, A., Bussoletti, E., Fonti, S., Palumbo, P., \& Mertins, H. C. 1995a, ApJ, 444, 288

Mennella, V., Colangeli, L., Bussoletti, E., Monaco, G., Palumbo, P., \& Rotundi, A. $1995 \mathrm{~b}, A p J S, \mathbf{1 0 0}, 149$

Stephens, J. R., Blanco, A., Bussoletti, E., Colangeli, L., Fonti, S., Mennella, V., \& Orofino, V. 1995, Planet Spa. Sci., 43, in press

Tokunaga, A. T., \& Brooke, T. Y. 1990, Icarus, 86, 208 